\title{
Editorial: Putting the Spotlight on the Role of Oxygen in Critically-III Patients: From Basic Mechanisms to Clinical Effects
}

\author{
Stefano Busani ${ }^{1 *}$, Elena Munari ${ }^{1}$, Fabio Silvio Taccone ${ }^{2}$, Abele Donati ${ }^{3,4}$ and \\ Massimo Girardis ${ }^{1}$ \\ ${ }^{1}$ Anesthesia and Intensive Care Unit, University Hospital of Modena Policlinico, University of Modena and Reggio Emilia, \\ Modena, Italy, ${ }^{2}$ Department of Intensive Care, Hôpital Erasme, Université Libre de Bruxelles (ULB), Bruxelles, Belgium, \\ ${ }^{3}$ Anesthesia and Intensive Care Unit, Azienda Ospedaliera Universitaria Ospedali Riuniti, Ancona, Italy, ${ }^{4}$ Department of \\ Biomedical Sciences and Public Health, Università Politecnica delle Marche, Ancona, Italy
}

Keywords: hyperoxia, hyperoxemia, ICU, critically-ill patients, children
OPEN ACCESS

Edited and reviewed by: Jlhad Mallat,

Cleveland Clinic Abu Dhabi, United Arab Emirates

*Correspondence: Stefano Busani stefano.busani@unimore.it

Specialty section: This article was submitted to Intensive Care Medicine and Anesthesiology,

a section of the journal

Frontiers in Medicine

Received: 21 January 2022 Accepted: 04 February 2022 Published: 25 February 2022

Citation: Busani S, Munari E, Taccone FS, Donati A and Girardis M (2022) Editorial: Putting the Spotlight on the Role of Oxygen in Critically-III Patients:

From Basic Mechanisms to Clinical

Effects. Front. Med. 9:859632.

doi: 10.3389/fmed.2022.859632
Editorial on the Research Topic

Putting the Spotlight on the Role of Oxygen in Critically-Ill Patients: From Basic Mechanisms to Clinical Effects

Oxygen is vital for life, but its liberal administration sometimes occurs in hospitals without evident pathological prerequisites. Exposure to high oxygen concentrations can potentially enhance the production of reactive oxygen species (ROS), which, if on one hand appear necessary for host defense, on the other hand may result harmful in some acute conditions $(1,2)$. In recent years, the possible complications related to excessive oxygen administration have been investigated (3); however, in critically ill patients, the critical threshold resulting in subsequent oxygen toxicity remains unclear. The aim of the Research Topic of the articles in this issue, dedicated to critically ill patients, was to outline some interesting issues on this Research Topic, starting from the molecular mechanisms up to different clinical applications. Five articles were submitted to this thematic collection, two of which were basic research, two mini-reviews, and one original research study.

In the preclinical study by Damiani et al. the physiological response to hyperoxia, normoxia, and mild hypoxia was evaluated in spontaneously breathing rats. The authors aimed to evaluate the effects of different inhaled oxygen fractions $\left(\mathrm{FiO}_{2}\right)$ on macrohemodynamics and microvascular perfusion. Results from this experiment showed that the exposure to hyperoxia was characterized by a transient increase in mean arterial pressure (MAP) and systemic vascular resistance (SVR), suggesting ongoing vasoconstriction. On the other hand, mild hypoxia was associated with a reduction in MAP and SVR, with no significant changes in cardiac index. When microcirculation was assessed at the muscular level, the exposure to hypoxia led to a vasodilatory response with an increase in the density of microvascular vessels without modification of the microvascular flow capacity, while microvascular density decreased under hyperoxia.

In contrast, another preclinical study by Li et al. focused on the role of hyperoxia on gut and microbiome injury in mice. In particular, the authors analyzed the time- and the dose-dependent effects of hyperoxia on the gut, using a transcriptome analysis to understand the mechanism of oxygen-induced gut injury related to innate immunity. The mice were randomly assigned to normoxia (control group, room air $\mathrm{O}_{2}$ ) or one of nine hyperoxia groups using different $\mathrm{FiO}_{2}$ during different temporal exposure. The results of this study showed that hyperoxia causes time- and dose-dependent gut injury characterized by mucosal 
atrophy (i.e., villus shortening), enterocyte death, intestinal barrier dysfunction, increase in pro-inflammatory cytokines, and reduction of anti-inflammatory cytokines. The authors proposed the hypothesis of the potential crosstalk between gut dysbiosis, and gut injury induced by hyperoxia. Indeed, inhaled oxygen might promote the expansion of oxygentolerant Enterobacteriaceae, which release bacterial lipopolysaccharides (LPS) through impaired tight junctions into the bloodstream, resulting in endotoxemia. LPS from gram-negative bacteria and peptidoglycan from gram-positive bacteria activate innate immunity (Toll-like receptor 4, Nod-like receptor P3, Nucleotide-binding oligomerization domain signaling pathways) leading to gut inflammation and cell death.

The two mini reviews focused on the effects of hyperoxemia in children and hyperoxia in the perioperative setting of adult patients. Pelletier et al. analyzed 12 studies on the association between hyperoxemia and outcomes in critically ill pediatric patients. Six of 12 studies considered heterogeneous patients admitted to pediatric intensive care units (ICUs), highlighting a $U$-shape relationship between oxygen and mortality; among these studies, four showed an increase in mortality with $\mathrm{PaO}_{2}$ $>300-400 \mathrm{mmHg}$, and only one $>550 \mathrm{mmHg}$. Four other studies focused on hyperoxemia in pediatric ICU patients after cardiac arrest; of these, only one demonstrated that hyperoxemia was associated with increased mortality, while the other three did not. A single retrospective study included ICU patients with severe traumatic brain injury, and it showed that $\mathrm{PaO}_{2}$ admission of $300-500 \mathrm{mmHg}$ was associated with increased survival at discharge. However, this study enrolled patients with concomitant chest trauma, which may be a confounding factor on the beneficial effect of increased $\mathrm{PaO}_{2}$ in traumatic brain injury. The optimal cut-off at which hyperoxemia was significantly increase the risk of harmful effects remained unclear; according to these studies, this cut-off ranged between 250 and $400 \mathrm{mmHg}$, demonstrating as well that it may subsist a polynomial "U-curve" relationship between $\mathrm{PaO}_{2}$ and mortality in pediatric critically ill patients, with both hypoxemia and hyperoxemia associated with increased mortality. Busani et al. described the pathophysiological effects of hyperoxia on different organs in the surgical setting. The Research Topic is somewhat controversial because the World Health Organization guidelines still recommend the administration of $80 \% \mathrm{FiO}_{2}$ to reduce surgical site infections in adult patients undergoing general anesthesia; on the other hand, growing pathophysiological evidence showed that hyperoxia causes deleterious effects on many organs. An elevated systemic oxygen supply could induce oxidative stress with inflammation, vasoconstriction, impaired microcirculation, activation of haemostasis, acute and chronic lung injury, coronary blood flow disturbances, cerebral ischemia, gut dysbiosis, and altered antibiotics susceptibility. Regarding the prevention of surgical wound infections, it has been demonstrated that ROS alter the actin cytoskeleton of macrophages and their antibacterial function. For all these reasons, the authors suggested that the maintenance of $\mathrm{PaO}_{2}$ within physiological limits during the perioperative process might be the best option.

Finally, Justus et al. conducted an exploratory, singlecenter, retrospective cohort study on critically ill patients on extracorporeal veno-arterial membrane oxygenation (VAECMO). The authors focused on the association between oxygenation, removal of carbon dioxide $\left(\mathrm{PaCO}_{2}\right)$ and mechanical ventilation setting with in-hospital mortality. Fifty-two patients were included in the analysis and $50 \%$ were alive at the time of hospital discharge. Compared with non-survivors, hospital survivors had a significantly lower ECMO blood flow rate and received significantly higher native lung minute ventilation. Hospital survivors had significantly lower cumulative mean $\mathrm{PaO}_{2}$ than non-survivors (117 vs. $154 \mathrm{mmHg}, p=0.04$ ). Hospital survivors also had significantly higher cumulative mean $\mathrm{PaCO}_{2}$ than hospital non-survivors. However, on multivariable logistic regression analysis, hyperoxemia, $\mathrm{PaCO}_{2}$, and minute ventilation were not independently associated with in-hospital mortality. The authors highlighted that hyperoxemia was common in this cohort mainly in the first $48 \mathrm{~h}$ of treatment, however their data did not show increased mortality in contrast to other reports that showed independent higher mortality. Rather, they concluded that interactions between hyperoxemia, $\mathrm{PaCO}_{2}$, and minute ventilation was more relevant than their individual association with survival.

Hence, in conclusion, the data and reviews published in this Research Topic have shown that hyperoxia is burdened by a series of deleterious effects in critically ill patient. However, it is not possible to make any kind of recommendation as the evidence are not yet conclusive on this debated Research Topic.

\section{AUTHOR CONTRIBUTIONS}

$\mathrm{SB}, \mathrm{EM}$, and FT revised the articles belonging to the Research Topics and wrote the original version of the Editorial. AD and MG revised the Editorial. All authors have contributed to the manuscript. All authors have read and approved the final version of the manuscript.

\section{REFERENCES}

1. Girardis M, Busani S, Damiani E, Donati A, Rinaldi L, Marudi A, et al. Effect of conservative vs. conventional oxygen therapy on mortality among patients in an intensive care unit: the oxygen-ICU randomized clinical trial. J Am Med Assoc. (2016) 316:1583-9. doi: 10.1001/jama.2016. 11993

2. Chu DK, Kim LH, Young PJ, Zamiri N, Almenawer SA, Jaeschke $\mathrm{R}$, et al. Mortality and morbidity in acutely ill adults treated with liberal vs. conservative oxygen therapy (IOTA): a systematic review and meta-analysis. Lancet. (2018) 391:1693-705. doi: 10.1016/S0140-6736(18 30479-3

3. Singer M, Young PJ, Laffey JG, Asfar P, Taccone FS, Skrifvars MB, et al. Dangers of hyperoxia. Crit Care. (2021) 25:440. doi: 10.1186/s13054-021-03815-y 
Conflict of Interest: The authors declare that the research was conducted in the absence of any commercial or financial relationships that could be construed as a potential conflict of interest.

Publisher's Note: All claims expressed in this article are solely those of the authors and do not necessarily represent those of their affiliated organizations, or those of the publisher, the editors and the reviewers. Any product that may be evaluated in this article, or claim that may be made by its manufacturer, is not guaranteed or endorsed by the publisher.

Copyright (C) 2022 Busani, Munari, Taccone, Donati and Girardis. This is an openaccess article distributed under the terms of the Creative Commons Attribution License (CC BY). The use, distribution or reproduction in other forums is permitted, provided the original author(s) and the copyright owner(s) are credited and that the original publication in this journal is cited, in accordance with accepted academic practice. No use, distribution or reproduction is permitted which does not comply with these terms. 